\title{
Probabilistic exact cloning and probabilistic no-signalling
}

\author{
Arun Kumar Pati \\ Quantum Optics and Information Group, \\ SEECS, Dean Street, University of Wales, Bangor LL 57 IUT, UK
}

(August 7, 2018)

\begin{abstract}
We show that non-local resources cannot be used for probabilistic signalling even if one can produce exact clones with the help of a probabilistic quantum cloning machine (PQCM). We show that PQCM cannot help to distinguish two statistical mixtures at a remote location. Thus quantum theory rules out the possibility of sending superluminal signals not only deterministically but also probabilistically. We give a bound on the success probability of producing multiple clones in an entangled system.
\end{abstract}

All the useful information that can be transmitted has a universal speed limit, namely, the speed of light. In quantum mechanics the situation seems to be changed when Einstein, Podolsky and Rosen put forward their famous thought experiment on two systems that had interacted in the past but are no longer in direct contact [1]. These kind of systems are described by entangled states which show non-local correlations that cannot be explained by any local hidden variable theory [2]. In [国] they proved that the measurement outcome of one system can instantaneously affect the result of the other. This suggested that one can exploit the non-local nature of the entangled states to send superluminal signals. However, it was shown that since the operators at space-like separated distance commute, the averages of the observable at distant site remain the same and do not depend on the operations carried out by the other (distant) party [3:4].

Subsequently, Herbert [5] put forward the idea of amplifying quantum states on one part of the system and performing a single measurement on many copies to get information about what the other party has done. It was then demonstrated by Wooters-Zurek [6] and Dieks [7] that such a multiplying device cannot exist due to linearity of quantum theory. This is now known as the "no-cloning theorem". Exact cloning of an unknown quantum state allows one to copy one part of an entangled system many times, which would give the possibility to find out what observable the other party has measured. This provides us with the means to communicate instantaneously. Since deterministic and exact cloning is ruled out by linear process, the superluminal signalling using non-local nature of entangled states was doomed. Further, it was found that it is the unitarity of quantum evolutions [8] which prevents us from cloning two non-orthogonal states exactly and this has been used against measuring a single quantum state [9]. In recent years various cloning machines which produce inaccurate copies [10,11] by unitary processes are conceived, but they cannot be used for superluminal signalling. In fact, Gisin [12] has shown that the fidelity criterion based on "no-signalling" 
and inaccurate copying are consistent with each other. The no signalling constraint has been used to generate optimal asymmetric clones [13].

A recent proposal by Duan and Guo [14] shows that two non-orthogonal states can be cloned exactly using unitary and measurement operations with a postselection. Furthermore, they [15] have shown that a set of states chosen secretly can be exactly cloned by a probabilistic cloning machine if and only if the states are linearly independent. Hardy and Song [16] have used no-signalling condition for the probabilistic cloning machine to find a limit on the number of state that can be cloned in a given Hilbert space of a quantum system. The exact quantum cloning have been studied from a state discrimination view by Chefles and Barnett [17]. They have also studied the interpolation between the inaccurate and exact cloning and proposed a network to implement the cloning operation [18]. Recently, we [19] have shown that the unitarity of quantum theory allows us to have a linear superposition of multiple exact clones along with a failure term iff the non-orthogonal states chosen secretly are linearly independent. This "novel cloning" machine is quite general one and all the probabilistic and deterministic cloning machines can be regarded as a special case of the "novel cloning" machine. We have also proposed a protocol which produces exact clones of an arbitrary qubit (universal cloning) with the help of classical communication and the use of entanglement [20].

It is known that if the quantum states can be cloned exactly then superluminal signals can be sent definitely. The question we address in this letter is whether exact probabilistic cloning allows us to send superluminal signals probabilistically. If a message can be transmitted instantaneously with certain non-zero probability less than unit, we call it probabilistic superluminal signalling. We show that given a probabilistic cloning machine which produces exact clones one cannot send superluminal signals probabilistically. Whereas deterministic superluminal signalling is impossible, there was some doubt that quantum mechanics might admit probabilistic superluminal signalling with the help of a probabilistic cloning machine. We give a bound on the success rate of producing $M$ exact clones of one part of system using PQCM in a composite system. This result shows that again the quantum theory is in agreement with the principles of special relativity.

Suppose we have a singlet state consisting of two particles shared by Alice and Bob. The state is given by

$$
\begin{aligned}
& \left|\psi^{-}\right\rangle=\frac{1}{\sqrt{2}}\left(\begin{array}{lll}
0 & \left.1\rangle-\mid \begin{array}{ll}
1 & 0
\end{array}\right)
\end{array}\right) \\
& =\frac{1}{\sqrt{2}}\left(|\psi\rangle\left|\psi_{\perp}\right\rangle-\left|\psi_{\perp}\right\rangle|\psi\rangle\right),
\end{aligned}
$$

where $|\psi\rangle=\alpha|0\rangle+\beta|1\rangle$ and $\left|\psi_{\perp}\right\rangle=\beta^{*}|0\rangle-\alpha|1\rangle$ are mutually orthogonal spin states (or polarisations in case of photon). We call this basis $\left\{|\psi\rangle,\left|\psi_{\perp}\right\rangle\right\}$ as the qubit basis. Alice is in possession of particle 1 and Bob is in possession of particle 2. Alice can chose to measure the spin along the $x$ - or the $z$-axis. It is known that given a shared EPR state between Alice and Bob, then the measurement outcomes of Bob are invariant under arbitrary local unitary transformation done by Alice. This is the basis for no-superluminal communication. The measurement outcomes of Bob is

$$
p(a)=\operatorname{tr}_{B}\left(\rho_{B} P_{a}\right)
$$


where the set of operator $\left\{P_{a}\right\}$ are projectors or could be some generalised measurements such as POVMs satisfying $\sum P_{a}^{\dagger} P_{a}=I$. The density matrix $\rho_{B}=\operatorname{tr} \rho_{A B}=\operatorname{tr}\left[\left(U_{A} \otimes\right.\right.$ $\left.\left.I_{B}\right) \rho_{A B}\left(U_{A} \otimes I_{B}\right)^{\dagger}\right]$ is invariant under a unitary operation by Alice. Hence Bob cannot distinguish two statistical mixtures resulting at his location due to the unitary operation done at a remote place. However, if Bob can produce exact clones (of any arbitrary input state) of his particle then he can distinguish two statistical mixtures. Is the same true with a probabilistic cloning machine?

For a single quantum system the probabilistic quantum cloning machine (PQCM) takes an input state $\left|\psi_{i}\right\rangle$ from a set $\mathcal{S}=\left\{\left|\psi_{i}\right\rangle\right\}(i=1,2, . ., K)$. This state is going to be cloned. Let $|A\rangle=|0\rangle^{\otimes M}$ an ancilla (a collection of $M$ blank states) and $\left|P_{0}\right\rangle,\left|P_{1}\right\rangle$ be the 'check' states which after a measurement tells us whether cloning has been successful or not. Following [15] it can be proved that there is a unitary transformation $U$ such that the following evolution holds

$$
\begin{aligned}
& \left|\psi_{i}\right\rangle|A\rangle\left|P_{0}\right\rangle \rightarrow U\left(\left|\psi_{i}\right\rangle|A\rangle\left|P_{0}\right\rangle\right)= \\
& \sqrt{p}_{i}\left|\psi_{i}\right\rangle^{\otimes M}\left|P_{0}\right\rangle+\sqrt{1-p_{i}}\left|\Phi_{i}\right\rangle\left|P_{1}\right\rangle
\end{aligned}
$$

if and only if the states $\left\{\left|\psi_{i}\right\rangle\right\}$ are linearly independent. Here, $p_{i}$ is the probability of successful cloning $M$ states and $\left|\Phi_{i}\right\rangle$ is the composite state of the input and blank states and these states need not be orthonormal. The unitary evolution together with a projection measurement yields $1 \rightarrow M$ exact clones with a probability of success $p_{i}$. To investigate the question of the possibility of the probabilistic superluminal signalling we carry out the action of PQCM on one part of a composite system (say) on Bob's particle 2. Bob attaches ancillas $C$ and $D$ and the evolution of the combined state is given by

$$
\begin{aligned}
& \left|\psi^{-}\right\rangle_{A B}|A\rangle_{C}\left|P_{0}\right\rangle_{D} \rightarrow U_{B C D}\left(\left|\psi^{-}\right\rangle_{A B}|A\rangle_{C}\left|P_{0}\right\rangle_{D}\right)= \\
& \frac{1}{\sqrt{2}}\left(\sqrt{p_{\perp i}}\left|\psi_{i}\right\rangle_{A}\left|\psi_{\perp i}\right\rangle_{B C}^{\otimes M}-\sqrt{p_{i}}\left|\psi_{\perp i}\right\rangle_{A}\left|\psi_{i}\right\rangle_{B C}^{\otimes M}\right)\left|P_{0}\right\rangle_{D} \\
& +\frac{1}{\sqrt{2}}\left(\sqrt{1-p_{\perp i}}\left|\psi_{i}\right\rangle_{A}\left|\Phi_{i}\right\rangle_{B C}-\sqrt{1-p_{i}}\left|\psi_{\perp i}\right\rangle_{A}\left|\tilde{\Phi}_{i}\right\rangle_{B C}\right)\left|P_{1}\right\rangle_{D},
\end{aligned}
$$

where the set $\left\{\left|\psi_{i}\right\rangle\right\}$ and $\left\{\left|\psi_{\perp i}\right\rangle\right\},(i=1,2)$ are linearly independent. The index $i$ refers to two possible choices of basis onto which Alice might do a measurement. For example, $\left\{\left|\psi_{i}\right\rangle\right\}=\{|0\rangle, \cos \theta|0\rangle+\sin \theta|1\rangle\}$ and $\left\{\left|\psi_{\perp i}\right\rangle\right\}=\{|1\rangle, \sin \theta|0\rangle-\cos \theta|1\rangle\}$. Bob performs a measurement on the probing device $P$ of the cloning machine. He keeps the states if the outcome is $\left|P_{0}\right\rangle$ and discards the result if the outcome is $\left|P_{1}\right\rangle$. From Eq.(4) it is clear that after postselection of measurement result the antisymmetric Bell state becomes

$$
\begin{aligned}
& \frac{1}{\sqrt{2}}\left(\left|\psi_{i}\right\rangle\left|\psi_{\perp i}\right\rangle-\left|\psi_{\perp i}\right\rangle\left|\psi_{i}\right\rangle\right) \rightarrow \\
& \frac{1}{\sqrt{2}}\left(\sqrt{p_{\perp i}}\left|\psi_{i}\right\rangle\left|\psi_{\perp i}\right\rangle^{\otimes M}-\sqrt{p_{i}}\left|\psi_{\perp i}\right\rangle\left|\psi_{i}\right\rangle^{\otimes M}\right) .
\end{aligned}
$$

If Bob can follow the above procedure, then the after Alice finds her particle in the basis $\{|0\rangle,|1\rangle\}$ the reduced density matrix of the particle 2 (with ancillas) will be

$$
\rho_{B C}=\operatorname{tr}_{D A}\left(\rho_{A B C D}\right)=\frac{1}{2}\left(p_{0}|0\rangle\left\langle\left. 0\right|^{\otimes M}+p_{1} \mid 1\right\rangle\left\langle\left. 1\right|^{\otimes M}\right) .\right.
$$


On the other hand if Alice finds her particle in the basis $\left|\psi_{2}\right\rangle=\cos \theta|0\rangle+\sin \theta|1\rangle,\left|\psi_{\perp 2}\right\rangle=$ $\sin \theta|0\rangle-\cos \theta|1\rangle\}$, then the reduced density matrix of the joint system $(B C)$ would be

$$
\rho_{B C}=\operatorname{tr}_{D A}\left(\rho_{A B C D}\right)=\frac{1}{2}\left(p_{2}\left|\psi_{2}\right\rangle\left\langle\left.\psi_{2}\right|^{\otimes M}+p_{\perp 2} \mid \psi_{\perp 2}\right\rangle\left\langle\left.\psi_{\perp 2}\right|^{\otimes M}\right) .\right.
$$

Since the two statistical mixtures in (6) and (7) are different this would have allowed Bob to distinguish two preparation stages by Alice, thus allowing for superluminal signalling probabilistically. However, this is not possible. Since there are four states involved in the cloning process and Bob's particle belong to a two-dimensional Hilbert space only two of them can be linearly independent. It can be seen from the form of equation (5) that $\left\{\left|\psi_{2}\right\rangle\right\}$ and $\left\{\left|\psi_{\perp 2}\right\rangle\right\}$ must be linearly dependent on the states $\left\{\left|\psi_{1}\right\rangle\right\}$ and $\left\{\left|\psi_{\perp 1}\right\rangle\right\}$. This means Bob's cloning machine should be able to clone the states from the set $\{|0\rangle,|1\rangle, \cos \theta|0\rangle+$ $\sin \theta|1\rangle, \sin \theta|0\rangle-\cos \theta|1\rangle\}$. But this set is linearly dependent, hence Bob cannot clone all these four states exactly with a non-zero probability. Thus there is no way for Bob to make an inference based on the measurement results of his $M$ clones and to know what Alice has done. Thus there cannot be any superluminal signalling even with a non-zero probability less than unit.

The no-signalling constraint leads to the observation by Hardy and Song [16] that one cannot clone more than certain number of linear independent states via a PQCM. Here, we derive a bound on the success rate of producing $M$ clones for $N$ linearly independent states for a composite system. Let us consider an arbitrary composite system whose state is described by

$$
|\psi\rangle_{A B}=\sum_{i j=1}^{N_{A} N_{B}} a_{i j}\left|x_{i}\right\rangle\left|y_{j}\right\rangle,
$$

where $\left\{\left|x_{i}\right\rangle\right\} \in \mathcal{H}_{A}=C^{N_{A}}$ and $\left\{\mid y_{j}\right\} \in \mathcal{H}_{B}=C^{N_{B}}$. Using the Schimdt decomposition we can write the bipartite state as

$$
|\psi\rangle_{A B}=\sum_{k=1}^{N} c_{k}\left|\alpha_{k}\right\rangle\left|\beta_{k}\right\rangle,
$$

where we have put $N_{A}=N$, where $N$ is the dimension of the smallest Hilbert space. Though Hilbert space $\mathcal{H}_{B}$ has $N_{B}$ number of linearly independent states in principle, only $N$ of them can be cloned exactly [16]. We can write the bipartite state as [21]

$$
|\psi\rangle_{A B}=\frac{1}{\sqrt{N}} \sum_{k=1}^{N}\left|u_{k}\right\rangle\left|v_{k}\right\rangle,
$$

where $\left|u_{i}\right\rangle$ are orthogonal basis for $\mathcal{H}_{A}$ and $\left|v_{i}\right\rangle$ are non-orthogonal and linearly independent basis states for $\mathcal{H}_{B}$. Suppose the subsystem $B$ is passed through a PQCM, then we have

$$
\begin{aligned}
& |\Psi\rangle_{A B C D}=U_{B C D}\left(|\psi\rangle_{A B}|A\rangle\left|P_{0}\right\rangle\right) \\
& =\frac{1}{\sqrt{N}} \sum_{k=1}^{N}\left[\sqrt{p_{k}}\left|u_{k}\right\rangle\left|v_{k}\right\rangle^{\otimes M}\left|P_{0}\right\rangle+\sqrt{1-p_{k}}\left|u_{k}\right\rangle\left|\Phi_{k}\right\rangle\left|P_{1}\right\rangle\right]
\end{aligned}
$$


After a postselction of measurement result the machine will yield the state

$$
|\tilde{\Psi}\rangle_{A B C}=\frac{1}{\sqrt{N}} \sum_{k=1}^{N} \sqrt{p_{k}}\left|u_{k}\right\rangle\left|v_{k}\right\rangle^{\otimes M} .
$$

We would like to know what is the success rate of producing $M$ clones of two distinct states at Bob's site chosen from $N$ linearly independent states. Let us define a basis in the Hilbert space $\mathcal{H}_{B C D}$ as $\left|X_{i}\right\rangle$, given by (using eq.(11) )

$$
\left.\left|X_{i}\right\rangle=\left\langle u_{i} \mid \Psi\right\rangle_{A B C D}=\frac{1}{N}\left[\sqrt{p_{i}}\right\rangle\left|v_{i}\right\rangle^{\otimes M}\left|P_{0}\right\rangle+\sqrt{1-p_{i}}\left|\Phi_{i}\right\rangle\left|P_{1}\right\rangle\right]
$$

Now taking the inner product of the two distinct basis we can get

$$
N\left|\left\langle X_{i} \mid X_{j}\right\rangle\right| \leq \sqrt{p_{i} p_{j}}\left|\left\langle v_{i} \mid v_{i}\right\rangle\right|^{M}+\sqrt{\left(1-p_{i}\right)\left(1-p_{j}\right)}\left|\left\langle\Phi_{i} \mid \Phi_{j}\right\rangle\right| .
$$

Simplifying the above inequality we have

$$
\frac{1}{2}\left(p_{i}+p_{j}\right) \leq \frac{\left(1-N\left|\left\langle X_{i} \mid X_{j}\right\rangle\right|\right)}{\left(1-\left|\left\langle v_{i} \mid v_{j}\right\rangle\right|^{M}\right)}
$$

The success probability in the case of composite system depends on the overlap of the actual states being cloned and also on the overlap of two non-orthogonal states belonging to a larger Hilbert space. In the limit of infinite number of cloning (when $M \rightarrow \infty$ ), this bound approaches some kind of state discrimination bound for a composite system, given by

$$
P=\frac{1}{2}\left(p_{i}+p_{j}\right) \leq\left(1-N\left|\left\langle X_{i} \mid X_{j}\right\rangle\right|\right)
$$

The maximum total success probability depends on the number of linearly independent states that can be cloned.

In conclusion, we have shown that even though a probabilistic quantum cloning machine can produce exact clones with certain non-zero probability still it is impossible to send superluminal signals with certain non-zero probability. Thus in the quantum theory the nosignalling condition is more stringent than that of the classical theory. In classical relativity we do not have a deterministic (with unit probability) superluminal signalling. But the quantum theory says that we cannot even have a probabilistic (less than unit) superluminal signalling. However, Kent 22] has shown that in a different quantum theory (in the context of time neutral cosmology) there is a superluminal signalling with non-zero probability.

I thank P. Kok, S. L. Braunstein and L. M. Duan for useful discussions. I thank L. Hardy for useful correspondence. The financial support from ESPRC is gratefully acknowledged. 


\section{REFERENCES}

[1] A. Einstein, B. Podolsky and N. Rosen, Phys. Rev. 47, 777 (1935).

[2] J. S. Bell, Speakable and Unspeakable in Quantum Mechanics, (Cambridge University Press, Cambridge, 1988).

[3] P. Eberhard, Nuovo Cim. 46 B, 392 (1978).

[4] G. C. Ghiradi, A. Rimini, and T. Weber, Lett. Nuovo Cim 27, 293 (1980).

[5] N. Herbert, Found. Phys. 12, 1171 (1982).

[6] W. K. Wootters and W. H. Zurek, Nature 299, 802 (1982).

[7] D. Dieks, Phys. Lett. A 92, 271 (1982).

[8] H. Yuen, Phys. Lett. A, 113, 405 (1986).

[9] G. M. D'Ariano and H. P. Yuen, Phys. Rev. Lett. 76, 2832 (1996).

[10] V. Bužek, M. Hillery, Phys. Rev. A 54, 1844 (1996).

[11] D. Bruß, D. P. Vincenzo, A. Ekert, C. Macchiavello and J. A. Smolin, Phys. Rev. A 57, 2368 (1998).

[12] N. Gisin, Phys. Lett. A 242, 1 (1998).

[13] S. Ghosh, G. Kar and A. Roy, Optimal Cloning and No Signalling, quant-ph/9907001.

[14] L. M. Duan and G. C. Guo, Phys. Lett. A 243, 261 (1998).

[15] L. M. Duan and G. C. Guo, Phys. Rev. Lett. 80, 4999 (1998).

[16] L. Hardy and D. D. Song, No Signalling and Probabilistic Cloning, quant-ph/9905024.

[17] A. Chefles and S. M. Barnett, J. Phys. A 31, 10097 (1998).

[18] A. Chefles and S. M. Barnett, Phys. Rev. A 60, 136 (1999).

[19] A. K. Pati, Quantum superposition of multiple clones and the novel cloning machine, quant-ph/9903038.

[20] A. K. Pati, Phys. Rev. A (1999) (in press).

[21] A. Chefles, Phys. Lett. A 239, 339 (1998).

[22] A. Kent, Phys. Rev. D 59, 43505 (1999). 